\title{
THE MAGNETIC MOMENT OF THE NEUTRINO AND ITS IMPLICATIONS FOR NEUTRINO SIGNAL FROM SN1987A
}

\author{
R. BARBIERI ${ }^{\text {a.b }}$, R.N. MOHAPATRA ${ }^{\mathrm{c}, 1}$ and T. YANAGIDA d.e \\ a Dipartimento di Fisica, Universitá di Pisa, I-56100 Pisa, Italy \\ INFN, Sezione di Pisa, Pisa, Italy \\ Department of Physics and Astronomy, University of Maryland, College Park, MD 20742, USA \\ " Randall Laboratory of Physics, University of Michigan, Ann Arbor, MI 48109. USA \\ Tohuku University, Sendai 980, Japan
}

Received 5 July 1988

\begin{abstract}
If the magnetic moment of the neutrino is to provide a resolution of the solar neutrino puzzle without violating the constraints implied by SN1987A observations, there must exist non-standard interactions of the right-handed neutrinos, which are able to trap them in the supernova core. We study one class of such interactions mediated by a singly charged Higgs boson, already proposed to yield a large magnetic moment of the neutrino, and suggest how the same interaction could provide a detectable signal in future neutrino counting experiments, $\mathrm{e}^{+} \mathrm{e}^{-} \rightarrow v \bar{v} \gamma$.
\end{abstract}

1. Recently, Voloshin, Vysotskii and Okun [1] have suggested that a non-vanishing magnetic moment of the neutrino may be responsible for the deficit of solar neutrinos observed in the Davis experiment [2]. For standard values of the solar magnetic field, $B_{\odot}=1-10 \mathrm{kG}$, the value of the magnetic moment of the $v_{\mathrm{e}}$ required to explain the observed deficit is $\mu_{v_{\mathrm{e}}} \simeq(0.1-1) \times 10^{-10} \mu_{\mathrm{B}}$, where $\mu_{\mathrm{B}}$ denotes the Bohr magneton. The interest in this suggestion primarily resides in the fact that it can neatly explain a possible time correlation between the solar neutrino flux, as observed by Davis and co-workers and the solar activity.

There is, however, a problem associated with this value of the magnetic moment [3] ${ }^{\# 1}$. In the absence of exotic interactions of the neutrinos, such a large value of $\mu_{v_{c}}$ is ruled out by SN1987A observations $[5,6]$ primarily because the left-handed neutrinos can flip their helicity via their magnetic moment interactions with electrons and protons in the supernova core; then the high energy $v_{\mathrm{eR}}\left(E_{v}>100 \mathrm{MeV}\right)$ produced in this process have a large mean free path and

\footnotetext{
Work supported by a grant from the National Science Foundation.

\#1 For a qualitative discussion, see ref. [4].
}

will escape leading to rapid cooling of the core. Since the observed neutrino signal at the IMB and Kamiokande detectors account for almost all of the binding energy of the stellar core $\left(E \simeq 4 \times 10^{53} \mathrm{ergs}\right)$, any additional cooling mechanism must be restricted. This leads to an upper bound $\mu_{v} \leqslant 3 \times$ $10^{-12} \mu_{\mathrm{B}}$. If one further notes that the emitted high energy $v_{R}$ 's can undergo a further spin flip in the galactic magnetic field in transit to earth, they could give rise to high energy $v_{\mathrm{L}}$-scattering events in the IMB and Kamiokande detectors. The fact that none has been observed implies a more stringent bound of $\mu_{\nu} \leqslant 10^{-13} \mu_{\mathrm{B}}$. This would, then, appear to rule out the neutrino magnetic moment as a solution to the solar neutrino problem.

Exceptions to the above argument can arise if the $v_{R}$ has exotic interactions, in which case, as we show below, one can reconcile both the SN1987A observation and a large $\mu_{v_{c}}$ for a very narrow range of parameters. This can arise if $v_{R}$ has an interaction with electrons that can cause trapping of the emitted $v_{R}$ 's in the supernova core. An interaction that couples the $v_{\mathrm{R}}$ 's exclusively to electrons is one mediated by an SU(2) singlet, singly charged Higgs boson, denoted by $\eta^{+}$, as suggested in a recent paper [7]. In ref. [7], it has already been shown that the singlet charged 
Higgs boson can lead to a large magnetic moment of the neutrino. The neutrino mass, however, remains arbitrary in this scheme ${ }^{\# 2}$ and a fine-tuning has to be invoked in order for the mass not to exceed the experimental bound.

In this letter, we show that contraints of low energy physics allow the $\eta^{+}$-interactions to be strong enough to avoid the supernova bound on $\mu_{\mathrm{v}}$. At the same time these interactions must be close to giving rise to a detectable signal in $\mathrm{e}^{+} \mathrm{e}^{-} \rightarrow v \bar{v} \gamma$ experiments.

2. The model we discuss is an extension of the standard $\mathrm{SU}(2)_{\mathrm{L}} \times \mathrm{U}(1)_{Y} \times \mathrm{SU}(3)_{\mathrm{C}} \quad\left(\equiv \mathrm{G}_{\mathrm{std}}\right)$ model which includes the right-handed neutrino $v_{R}$ (one per generation) and a charged singlet Higgs boson, $\eta^{+}$ with quantum numbers $(0,2,1)$ under $G_{\text {std }}$. In addition to the interactions present in the standard model, this model allows for the following new interactions:

$$
\begin{aligned}
& \mathscr{L}_{Y}^{\prime}=h_{i j}^{v} \bar{\psi}_{\mathrm{L}}^{i} \tilde{\phi} v_{\mathrm{R}}^{j}+g_{i j} \psi_{\mathrm{L}}^{i \mathrm{~T}} c^{-1} \tau_{2} \psi_{\mathrm{L}}^{\prime} \eta^{+} \\
& \quad+f_{i j} \mathrm{e}_{\mathrm{R}}^{\mathrm{T}} c^{-1} v_{\mathrm{R}}^{\prime} \eta^{+}+\text {h.c. }
\end{aligned}
$$

In eq. (1), $\psi_{\mathrm{L}}$ denotes the left-handed leptonic doublet, $\phi \equiv\left(\begin{array}{c}\phi+ \\ \phi_{0}^{+}\end{array}\right)$(and $\tilde{\phi}=\mathrm{i} \tau_{2} \phi^{*}$ ) denotes the Higgs doublet of the standard model. First, we note that $g_{i j}=-g_{j i}$ and $f_{i j}$ is an arbitrary matrix. By redefinition of fields, we can write $f_{i j}$ in the following form without any loss of generality:

$f=\left(\begin{array}{lll}f_{11} & 0 & 0 \\ f_{21} & f_{22} & 0 \\ f_{31} & f_{32} & f_{33}\end{array}\right)$

The dominant contribution to the magnetic moment in this model arises from the $g$ and $f$ interactions and is given by [7]

$$
\begin{aligned}
& \left(\mu_{v}\right)_{i j}=\frac{e}{32 \pi^{2} M_{\eta}^{2}} \sum\left[\left(f^{*} m g^{\mathrm{T}}\right)_{i j}+\left(g^{*} m f^{\mathrm{T}}\right)_{i j}\right] \\
& \quad \times\left[\ln \left(M_{\eta}^{2} / m_{1}^{2}\right)-1\right]
\end{aligned}
$$

where $M_{\eta}$ denotes the mass of the $\eta^{+}$-boson, $m$ denotes the diagonal charged lepton mass matrix; $m=$ $\operatorname{Diag}\left(m_{c}, m_{\mu}, m_{\tau}\right)$. It is clear from eq. (3), that the

\footnotetext{
\#- For left-right symmetric extensions of this model that also yield finite one-loop neutrino mass [8]. In this case, there is a relation between neutrino mass and magnetic moment matrix, which suppresses the value of $\mu_{\mathrm{v}_{\mathrm{c}}}$ [9].
}

dominant contribution to $\left(\mu_{v}\right)_{11}$ will come from the $\tau$-lepton intermediate state and can, therefore, a priori be quite large.

There exist a variety of other constraints [11,12] on the couplings $f$ and $g$ implied by the low energy data, coming from $g-2, \mu \rightarrow \mathrm{e} \gamma$ and muon decay, which can all be satisfied by the following specific forms for $g$ and $f$ :

$g=\left(\begin{array}{ccc}0 & 0 & g_{13} \\ 0 & 0 & 0 \\ -g_{13} & 0 & 0\end{array}\right), \quad f=\left(\begin{array}{ccc}0 & 0 & 0 \\ 0 & 0 & 0 \\ f_{31} & 0 & f_{33}\end{array}\right)$.

The first point to note about this form is that it implies that only $\mu_{v_{13}}$ receives contributions from the new interactions. We define $v_{3 R}$ to be the right-handed partner of $v_{\mathrm{CL}}$. We further assume that all neutrino masses are vanishingly small. In order to obtain a large $\mu_{v_{\mathrm{c}}}$ needed to solve the solar neutrino puzzle, we must have

$g_{13} f_{33} \simeq(0.02-0.2) G_{\mathrm{F}} M_{\eta}^{2}$.

Furthermore, the $g$ and $f$ interactions will contribute to $\tau$-decay, so near $\mu$-e universality observed in $\tau$-decay as well as the Michel parameter will imply constraints on $g_{i j}$ and $f_{i j}$. These constraints are:

(i) from $\mu-\mathrm{e}$ universality [13] (using two standard deviations)

$g_{13}^{2} \leqslant 0.25 G_{\mathrm{F}} M_{\eta}^{2}$

(ii) from the Michel-parameter in $\tau$-decay (using one standard deviation )

$g_{13} f_{33} \leqslant 6 G_{\mathrm{F}} M_{\mathrm{\eta}}^{2}$,

all consistent with eq. (5).

Let us now see if in this model, the supernova constraints on $\mu_{\mathrm{v}_{\mathrm{e}}}$ can be avoided.

3. As briefly mentioned in the introduction, in the absence of $\eta^{+}$-interactions, the mean free path of the $v_{R}$ produced due to the magnetic moment is larger than the core radius, leading to rapid core cooling via $v_{R}$-emission, in conflict with observation. In the presence of $\eta^{+}$-interactions, on the contrary, $v_{R}$ can get trapped, if its interactions are strong enough. To discuss these questions, we need the strength of $v_{R} e$ interactions, which arise via $\eta^{+}$-exchange (note that $\left.v_{3 K} \equiv v_{\mathrm{cR}}\right)$ 


$$
\begin{aligned}
& H_{\mathrm{eff}}=\left(f_{31}^{2} / M_{\eta}^{2}\right) v_{\mathrm{cR}}^{\mathrm{T}} c^{-1} \mathrm{e}_{\mathrm{R}} \cdot \mathrm{e}_{\mathrm{R}}^{+} c v_{\mathrm{cR}}^{*} \\
& \quad+\left(f_{31} g_{13} / M_{\eta}^{2}\right) v_{\mathrm{eR}}^{\mathrm{T}} c^{-1} \mathrm{e}_{\mathrm{R}} \cdot \mathrm{e}_{\mathrm{L}}^{+} c v_{\mathrm{rL}}^{*}+\text { h.c. }
\end{aligned}
$$

The only constraints on $v_{\mathrm{cR}} \mathrm{e}$ interactions depicted above comes from neutrino counting experiments $\mathrm{e}^{+} \mathrm{e}^{-} \rightarrow v \bar{v} \gamma$. In the same way as it is used to contrain the mass of the supersymmetric scalar electron via the process $\mathrm{e}^{+} \mathrm{e}^{--} \rightarrow \tilde{\gamma} \tilde{\gamma} \gamma$ [14], this experiment also in view of eq. (6) requires,

$f_{31}^{2} \lesssim 6 G_{\mathrm{F}} M_{\eta}^{2}$.

Let us now turn to the trapping conditions for $v_{R}$ in the supernova. The discussion here is very similar to that in ref. [15]. Let us denote the $v_{R}$ e thermally averaged scattering cross section by $\left(E_{\mathrm{e}} \simeq E_{\mathrm{v}}\right)$

$\sigma_{\mathrm{vR}} \simeq A \cdot G_{\mathrm{F}}^{2} E_{\mathrm{v}}^{2}, \quad A=(1 / 16 \pi) f_{31}^{4} / G_{\mathrm{F}}^{2} M_{\eta}^{4}$.

As noted in ref. [ 15], for $A<10^{-6}$, the mean free path of $v_{\mathrm{R}}$ exceeds the radius of the supernova core. For $A>10^{-6}, v_{\mathrm{R}}$ gets trapped and we have to consider the radius, $R_{v_{K}}$ of the $v_{R}$-sphere. The temperature of the emitted $v_{R}$ 's is governed by the temperature at the surface of the $v_{R}$-sphere. Assuming that

$T(R)=T_{\mathrm{c}}\left(R_{\mathrm{c}} / R\right)^{m / 3}, \quad m=3-7$

and using the fact that $R_{V_{R}} \propto A^{3 /(5 m-3)}$, we can take the ratio of the $v_{\mathrm{L}}$ and $v_{\mathrm{R}}$ luminosities as follows:

$Q_{v_{\mathrm{K}}} / Q_{v_{\mathrm{L}}}=\left(T_{\mathrm{vR}_{\mathrm{R}}} / T_{v_{\mathrm{L}}}\right)^{4}\left(R_{v_{\mathrm{R}}} / R_{\mathrm{v}_{\mathrm{L}}}\right)^{2}$

and find

$Q_{\mathrm{v}_{\mathrm{R}}}=Q_{\mathrm{v}_{\mathrm{L}}} A^{-(4 m-6) /(5 m-3)}$.

A point worth emphasizing here is that according to eq. (13), the weaker the strength of $v_{R}$ interactions, the greater the $v_{R}$ luminosity. If we demand that the $v_{\mathrm{R}}$ luminosity does not exceed $10^{53} \mathrm{ergs} / \mathrm{s}$ or that $Q_{v_{\mathrm{K}}} \lesssim 20 Q_{\mathrm{vL}}$, we get $A \gtrsim 10^{-2}-10^{-3}$ for $m=3-7$. For the strength of $v_{\mathrm{R}}$ interactions, eq. (10) implies that,

$f_{31}^{2} \gtrsim(0.2-1) G_{\mathrm{F}} M_{\eta}^{2}$.

As a matter of fact the lower bound (14) is close enough to the upper bound (9), to make conceivable the search for the anomalous electron-neutrino interaction, eq. (8), in planned neutrino counting experiments, for example at TRISTAN. Notice that the elastic $v_{\mathrm{e}}$ scattering experiments are not sensitive to the interaction (8) because of the left-handed helic- ity of the neutrinos in available "neutrino-beams". Notice also that the $\mathrm{e}^{+} \mathrm{e}^{-} \rightarrow v \bar{v} \gamma$ experiments should be done at a CMS energy not too close to the Z-boson mass, in order to avoid dominance of the $z$-pole in the cross section.

A further reason to think that the bound (9) could actually be saturated is provided by the following considerations. As discussed in refs. $[3,4]$, the righthanded neutrinos and antineutrinos ${ }^{\# 3}$ emitted by the supernova might undergo a helicity flip in the galactic magnetic field of about a microgauss. This flipping is actually bound to occur, except that a small but non-vanishing chance exists that the oscillatory function describing the neutrino helicity along the path between the Magellan cloud and the earth, could find itself in a node when reaching the earth. Bearing this possibility, one would have on earth a large amount of flipped $v_{L}$ and $\bar{v}_{L}$, generally more energetic than the standard $v_{\mathrm{L}}, \bar{v}_{\mathrm{L}}$ directly produced in the supernova core. This is because the temperature $T_{\mathrm{vR}}$ of the $v_{\mathrm{R}}$-sphere, relative to the standard $T_{\mathrm{v}_{\mathrm{I}} \text {. }}$ scales as

$T_{\mathrm{vR}} / T_{\mathrm{vL}}=A^{-m /(5 m-3)}$.

Therefore, in order not to conflict with the IM and Kamiokande observations, if flipping occurs when the $v_{\mathrm{R}}$ 's and $\bar{v}_{\mathrm{R}}$ 's reach the earth, we must require $A$ not to be too small. Notice that $A \simeq 1$, from eq. (10), means that the bound (14) gets saturated. We think that one further effective neutrino unit, as would result from $A$ being close to 1 does not conflict with the standard nucleosynthesis picture. On the other hand, this calls for a detectable signal in future neutrino counting experiments.

One of us (T.Y.) would like to thank the University of Michigan high energy theory group for hospitality.

\footnotetext{
\#3 Notice that the reactions producing right-handed antineutrinos via $\eta^{+}$-exchange, $\mathrm{e}^{+} \mathrm{e}^{-} \rightarrow \bar{v}_{R} v_{R}$ or $\bar{v}_{\mathrm{L}} \mathrm{e} \rightarrow \bar{v}_{R} \mathrm{e}$, have a characteristic time much smaller than $1 \mathrm{~s}$. This means that also the $\bar{v}_{R}$ 's will be in equilibrium inside the $v_{R}$-sphere.
}

\section{References}

[1] M.B. Voloshin, M.I. Vysotskii and L.B. Okun, Zh. Eksp. Teor. Fiz. 91 (1986) 754 [Sov. Phys. JETP 64 (1986) 446]; see also: A. Cisneros, Astrophys. Space Se. 10 (1971) 87. 
[2] R. Davis Jr., Proc. Seventh Workshop on Grand unification (ICOBAN 1986, Toyama, Japan) (World Scientific, Singapore, 1986).

[3] R. Barbieri and R.N. Mohapatra, Phys. Rev. Lett. 61 (1988) 27 ;

J. Lattimer and J. Cooperstein, Phys. Rev. Lett. 61 (1988) 23;

D. Notholz, Max Planck Institute preprint MPI-PAE/PTH $15 / 88$.

[4] I. Goldman, Y. Aharanov, G. Alexander and S. Nussinov, Phys. Rev. Lett. 60 (1988) 1789.

[5] K. Hirata et al., Phys. Rev. Lett. 58 (1987) 1490.

[6] R. Bionta et al., Phys. Rev. Lett. 58 (1987) 1494.

[7] M. Fukugita and T. Yanagida, Phys. Rev. Lett. 58 (1987) 1807 ;

K.S. Babu and V.S. Mathur, Phys. Lett, B 196 (1987) 218.
[8] R.N. Mohapatra, Phys. Lett. B 201 (1988) 517.

[9] J. Pulido and J. Ralston, FermiLab preprint (1988); R.N. Mohapatra, Proc. INS Symp. on Neutrino mass and related topics, to be published.

[10] J. van der Velde, Talk XXIII Recontre de Moriond on Dark matter (Les Arcs, March 1988).

[11] M. Fukugita and T. Yanagida, Kyoto preprint RIFP-738 (1988).

[12] A. Zee, Phys. Lett. B 161 (1987) 141.

[13] Particle Data Table (1987).

[14] ASP Collab., C. Hearty et al., Phys. Rev. Lett. 58 (1987) 1711 ;

MAC Collab., E. Fernandez et al., Phys. Rev, Lett. 54 (1985) 1118.

[15] R. Barbieri and R.N. Mohapatra, University of Maryland preprint 88-205 (1988). 